\title{
REVISIÓN DE LA CAPACIDAD DE TRANSPARENCIA Y CONFIANZA QUE OFRECE LA TECNOLOGÍA BLOCKCHAIN
}

Manuela Linares Barbero malinare@ulima.edu.pe Universidad de Lima. Lima, Perú

\section{Resumen}

La confianza es un elemento fundamental del mundo contemporáneo, especialmente en las diferentes transacciones que realizan las empresas. El incremento del fraude, así como la falsificación de productos y servicios, han generado que las compañías deban gastar dinero y esfuerzos en reprocesos y medidas de seguridad. Las transacciones transparentes se han convertido un reto que muchos buscan lograr. Una de las grandes propuestas de transacciones confiables es la tecnología blockchain. Esta puede ofrecernos, transparencia en las transacciones y confianza entre las organizaciones, aun si no se conocen entre ellas previamente. En nuestro artículo desarrollaremos la revisión de la literatura de las capacidades de transparencia y confianza que nos presenta la tecnología blockchain en la cadena de suministro.

Palabras clave: blockchain, cadena de suministro, transparencia, confianza, confiabilidad, sistemas distribuidos

\section{Abstract}

\section{Reviewing the capacity for transparency and trust offered \\ by the blockchain technology}

Trust is a fundamental element of the contemporary world, especially in the different transactions carried out by companies. The increase of fraud, as well as the falsification of products and/or services, has generated that companies must spend money and efforts in implementing reprocesses and security measures. Transparent transactions have become a challenge that many seek to achieve. One of the great proposals of reliable transactions is the blockchain technology. It can offer us transparency in transactions and trust among organizations, even if they do not know each other beforehand. In our article, we will review the literature on the capacity for transparency and trust that the blockchain technology presents in the supply chain.

Keywords: blockchain, supply chain, transparency, trust, reliability, distributed systems 


\section{Introducción}

Existen serios problemas de falsificación y fraude en la cadena de suministro de las empresas. Muchas de estas reducen el problema en mención con tecnología RFID (Radio Frecuency Identification); sin embargo, las etiquetas que utiliza son fácilmente copiadas o modificadas por personas inescrupulosas. Tomando en cuenta que las empresas mantienen toda la información en un sistema centralizado, es decir, en una única ubicación, quedan expuestas a riesgo de robos, soborno al personal o pérdidas de información sensible de los productos $y$, muchas veces, de los datos de los clientes (Tian, 2017). Por este motivo, los consumidores han perdido la confianza en las compañías comerciales, con el temor de que los productos comprados se hayan adulterado o de que su información sea robada.

Para eliminar los problemas expuestos y sobre todo para ganar nuevamente la confianza de los clientes, contamos con la propuesta que nos trae la nueva plataforma blockchain. Se trata de una tecnología disruptiva que cambiará el mundo empresarial, así como lo hizo la imprenta en siglo xv y la internet en el siglo xx (Faye, 2017). Utilizando un sistema distribuido, logra mantener la integridad de la información y al mismo tiempo la difunde entre todos los participantes. La aplicación más conocida es la criptomoneda, como bitcoin, la cual tiene más del $23 \%$ de sus nodos en Estados Unidos (Yeow, 2018) y fue creada para reducir o eliminar el doble gasto en las transacciones (Christidis y Devetsikiotis, 2016), pero dicha tecnología no es exclusiva de ella.

La plataforma mencionada ofrece múltiples aplicaciones: en la cadena de suministro, en el sector de educación, en la sostenibilidad del medio ambiente, entre otros, las que pueden beneficiarse de las capacidades de trazabilidad, transparencia y confiabilidad.

Una aplicación interesante en la cadena de suministro es Everledger, que utiliza la tecnología blockchain para rastrear diamantes desde su extracción en bruto hasta el actual poseedor de la joya. Así se contribuye con la eliminación del mercado negro de diamantes (Kshetri, 2018).

Incorporando la propuesta de seguimiento de localización, identificamos las coordenadas exactas donde se encuentra cada objeto (Kamel Boulos, Wilson y Clauson, 2018). Observamos que mediante este sistema es posible colaborar con las empresas, pues se evitaría que estas proyecten una imagen negativa generada por el fraude o por productos alterados. Adicionalmente, lograría reducciones de tiempo y costos en las transacciones así como robos.

En nuestro artículo desarrollaremos la revisión de la literatura de las capacidades de transparencia y confianza que nos presenta la blockchain en lo que se refiere a la cadena de suministro. También exploraremos los beneficios en otras aplicaciones que se desarrollan con la plataforma mencionada.

En la figura 1 podemos apreciar las bases de datos de búsqueda y la selección que se realizó de los artículos encontrados, filtrando las 40 fuentes utilizadas en esta revisión. 


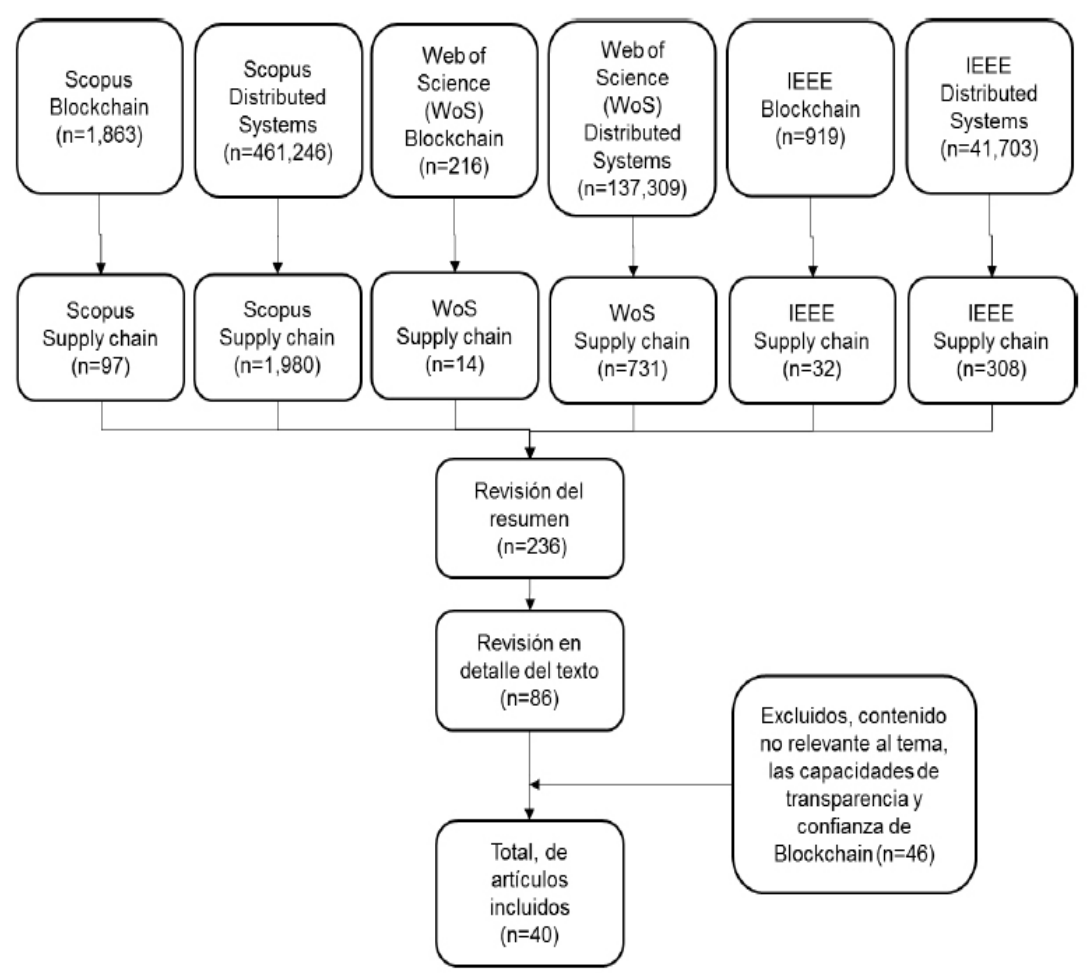

Figura 1. Estrategia de selección de artículos

Elaboración propia.

\section{Transparencia y confianza que ofrece blockchain: cadena de suministro}

\subsection{Modelo de negocio}

Apreciamos la creación de nuevos modelos de negocio como lo es DAO (Decentralized Autonomous Organization), una organización creada completamente con base en Smart Contracts (Atzei, Bartoletti y Cimoli, 2017). Con estos nuevos modelos de negocio se expanden los horizontes a un mundo totalmente descentralizado en el que las organizaciones no son controladas por unos pocos individuos sino por todos los interesados, es decir, todos los nodos en la red, los que, con su aprobación o rechazo de los bloques controlan las transacciones que se realizan en la organización. Este nuevo modelo de negocio nos conduce a un mundo empresarial que hace unos años parecía utópico, pero hoy con la tecnología de blockchain, combinada con el concepto de Smart Cities, a través de servicios compartidos (Sun, Yan y Zhang, 2016), nos demuestra que ya es una realidad.

No obstante, aún queda mucho camino por recorrer, y estas soluciones no están exentas de riesgos y problemas, como es el caso de DAO, desarrollado en la plataforma 
Ethereum y programada en Solidity, lenguaje de programación de la blockchain mencionada. En él se puede dar la pérdida de ether (token de la plataforma), al enviar a una dirección inválida, o puede sufrir ataques al DAO para robar los ethers. Ejemplo de ello fue el suceso del 18 de junio del 2016, cuando un atacante logró registrar 60 millones de dólares americanos en ethers bajo su control (Atzei, Bartoletti y Cimoli, 2017).

El caso mencionado nos demuestra la importancia de mantener las leyes en el mundo digital. El gran beneficio de tener un sistema distribuido consiste en evitar la centralización y los riesgos que trae consigo, como la mayor vulnerabilidad de ataques, los problemas de corrupción, como sobornos a quienes manejan la información y abusos de esta. Podemos ver propuestas de solución basadas en la generación de Smart Contracts para lograr la identificación por parte de los usuarios, asociándolos a su data o a un controlador específico, o por parte del controlador, vinculándolos al tipo de información sin importar el usuario (Neisse, Steri y Nai-Fovino, 2017). Las propuestas señaladas nos indican el camino para poder cumplir con las leyes de protección de datos en la plataforma blockchain, y así mantener la confianza de las organizaciones, los consumidores y la comunidad.

\subsection{Transparencia en la cadena de suministro con blockchain}

No es extraño que en el mundo comercial los consumidores sean víctimas de personas inescrupulosas que alteran los productos. A esta situación se añaden los riesgos a los que nos exponen el medio ambiente y el manejo de la mercancía en producción, empaque y distribución, especialmente medicamentos, alimentos y bebidas.

El medio ambiente se presenta como un factor crítico en el transporte de algunos bienes sensibles a la temperatura y la humedad, como los medicamentos. Para cumplir con la nueva regulación de la Unión Europea, las empresas de transporte de medicinas utilizan camiones refrigerantes en todas sus rutas. La start-up Modum, en colaboración con la Universidad de Zürich, diseñó un sistema para la distribución de medicinas dirigido a reducir el costo del transporte al minimizar el uso de camiones frigoríficos (Kshetri, 2018), los que evitan el deterioro de la mercadería. Los retos del medio ambiente están presentes en el transporte de los productos agrícolas, desde la cosecha hasta el supermercado y sus múltiples intermediarios. Estos manejan datos sensibles del traslado que ellos mismos realizan y de quien les entregó los productos; se pierde así información fundamental del estado real de los alimentos en otras partes de la cadena. Para evitar este problema, la tecnología blockchain nos puede apoyar eficazmente con la difusión de la información (Faye, 2017), y facilita la transparencia que requieren todos los involucrados en la red.

En la cadena de suministro se han de tomar en cuenta los siguientes factores: los costos, la velocidad, la dependencia, la reducción del riesgo, la sostenibilidad y la 
flexibilidad. Para este conjunto de elementos se han desarrollado varios proyectos piloto con la plataforma blockchain.

Algunos han sido combinados con la tecnología loT (Internet of Things o el internet de las cosas) (Kshetri, 2018). También existen propuestas que pretenden mejorar la transparencia e integridad de los datos usando la ayuda de sensores loT asegurados con claves públicas y privadas (Faye, 2017), manteniendo así los certificados de origen. Con dicha plataforma se ofrece al consumidor de forma transparente la información del producto, influenciando en gran medida su decisión de compra (Sander, Semeijn y Mahr, 2018).

En el intento de eliminar los riesgos de enfermedades que podrían sufrir los clientes por la ingesta de productos alimenticios, se han desarrollado diferentes modelos de control como HACCP (Hazard Analysis and Critical Control Points) de la FDA (Food and Drug Administration), que busca eliminar riesgos biológicos, químicos y físicos que puedan dañar los alimentos (Tian, 2017).

Ello sucedió en el 2015 con Chipotle Mexican Grill, al generarse un brote de Escherichia coli en 55 consumidores (Casey y Wong, 2017). El modelo de control HACCP se puede desarrollar con una plataforma privada de blockchain y así garantizar la confiabilidad de la información, como temperatura, humedad y otras características que es necesario monitorear durante el transporte y dar avisos a las tiendas o restaurantes de las fechas de vencimiento para eliminar la mercadería que pueda enfermar a sus clientes (Tian, 2017). De esta manera evitamos el desprestigio de la marca comercial y del establecimiento en el que se expenden los alimentos.

Los riesgos a los que se exponen los consumidores al adquirir productos en mal estado, especialmente alimentos y medicinas, se presentan como un reto que ha de enfrentarse inmediatamente. En lo que respecta a las medicinas, la FDA (Food and Drug Administration), con el Acta de Seguridad e Innovación (Safety and Innovation Act) y el Acta de Seguridad en la Cadena de Suministro de los Medicamentos (Drug Supply Chain Security Act) en los Estados Unidos, y la Directiva de Medicinas Falsificadas (Falsified Medicines Directive) en la Unión Europea, ofrecen normas para garantizar la vigilancia de los productos farmacéuticos (Beninger, 2017). Estas ayudan a eliminar el problema de medicamentos alterados $y$, recurriendo a la implementación de la tecnología blockchain en la cadena de suministro en todas sus etapas, a garantizar las normas de seguridad por medio de sensores en los laboratorios y en el transporte de insumos de cada fármaco, para evidenciar que no han sido alterados antes de la producción. Se deben medir los indicadores necesarios durante la producción de los medicamentos y después en su empaquetado, transporte y almacenamiento, hasta que llegan al consumidor en una farmacia, hospital o cualquier centro médico donde se atienda al paciente. La transparencia de la información que nos ofrece blockchain permite que cualquier entidad dentro de la cadena de suministro pueda rechazar medicamentos $o$ insumos que no cumplan los estándares determinados por las normas legales. 


\subsection{Confianza en la cadena de suministro con blockchain}

Cuando pensamos en la cadena de suministro de productos alimenticios, la confianza en la información que maneja la misma es de vital importancia, especialmente con los bienes perecibles de corta vida, los que necesitan mantenerse entre ciertas temperaturas. La utilización de una plataforma de blockchain con el uso de la tecnología loT, puede solucionar no solo el problema de trazabilidad, sino también el de la información relacionada al deterioro del bien. Existen varias propuestas para la combinación de estas tecnologías en la cadena de suministro, como es la de AgriBlockloT (Pincheira Caro, Salek Ali, Vecchio y Giaffreda, 2018), pruebas piloto de Walmart con IBM (Firica, 2017), en el mercado de segunda mano (Toyoda, Sasase, Ohtsuki y Mathiopoulos, 2017), en la planeación de la producción de aviones (Madhwal y Panfilov, 2017), en la geolocalización de la mercadería transportada (Wu, Li, King, Miled, Wassick y Tazelaar, 2017), el empleo de una plataforma privada con cierta información pública (Engelenburg, Janssen y Klievink, 2017), entre otras.

Todas enfrentan los mismos retos relacionados a la verificación del sensor y la información que emite. Qu, Tao, Zhang, Hong y Yuan (2018) nos ofrecen una solución basada en una estructura jerárquica de blockchain con nodos que gestionan el servicio. Los nodos del nivel inferior están conectados a los dispositivos loT, verifican su identidad, localización e integridad de información antes de crear el bloque, difundirlo en su nivel y enviarlo al nodo del siguiente nivel, el que repetirá el proceso hasta llegar al nivel superior. Con la propuesta mencionada se puede evitar que la información sea alterada maliciosamente o por defectos del sensor y pueda ingresar a la cadena de bloques.

Así como la verificación de los sensores loT es de vital importancia, también lo es la validación de los usuarios al momento de realizar transacciones. Cada organización tiene sus propios mecanismos para reconocer a sus clientes, especialmente si se realizan operaciones con alto valor monetario. Podemos identificar una propuesta para KYC (know-your-customer, conoce a tu cliente), que busca verificar al mismo usando la tecnología de registro distribuido que nos ofrece blockchain, en la que se valida solo una vez al consumidor y se comparte esta información con todas las organizaciones que la necesiten (Parra Moyano y Ross, 2017). Se reduce así el doble proceso que realiza cada organización al validar de manera independiente a sus compradores y se logra la transparencia de la información de manera eficiente.

\section{Algunos aspectos de seguridad que plantea el uso de blockchain}

\subsection{Posibles ataques}

El mayor beneficio que nos ofrece la tecnología blockchain es la seguridad de los datos a nivel de integridad como de confiabilidad, al ser un sistema distribuido en el que solo se 
pueden agregar bloques y no modificar o eliminar los mismos de la cadena. Sin embargo, esto no evita que personas maliciosas quiera alterar la cadena aprobando o rechazando bloques a su favor, generando mining pools, grupos de mineros, para obtener más del $51 \%$ del hash power (Lin y Liao, 2017). Con el ataque del $51 \%$ un nodo minero puede agregar bloques para generar doble gasto o robar tokens (Xu, 2016). Esto ha llevado a que más del $50 \%$ del hash power se ubique en China (Yeow, Gani, Rodrigues, Ahmad y Ko, 2017). Actualmente no se producen estos ataques porque el valor del token, la recompensa por el minado, es significativo en comparación con el valor de la transacción (Lin y Liao, 2017). Al problema en mención se añade el sybil attack (Yeow, Gani, Rodrigues, Ahmad y Ko, 2017) que genera múltiples identidades.

En la blockchain pública la identidad de los participantes es anónima. Si bien todos los nodos tienen acceso a ver las transacciones en la cadena de bloques, no se puede identificar a los participantes pues estos se identifican en la red con su clave privada. Tal situación genera el problema de robo de identidad. Si la clave privada es robada, el infractor tendrá acceso a robar todos los bienes y, al ser anónima, no habrá forma de identificarlo (Xu, 2016). Esto nos demuestra lo importante que es el desarrollo de nuevas políticas en cuanto a la identidad de los participantes en la red. Los ataques reseñados son propios de las blockchains públicas y no ocurren en las blockchains privadas o de consorcio porque en estas existe una lista predeterminada de nodos y sus accesos que hace innecesarios los incentivos.

\subsection{Problemas fork}

Algunos otros inconvenientes surgidos en las plataformas blockchain no se generan por intenciones maliciosas, como los problemas fork, que aparecen cuando dos nodos aprueban el bloque al mismo tiempo y dividen la cadena (Hanifatunnisa y Rahardjo, 2017). No es normal que dos nodos aprueben un bloque al mismo tiempo, sin embargo, cuando existen dos versiones de la plataforma en la misma red, los nodos validan bloques con protocolos de consenso diferentes y generan la bifurcación en la cadena (Kamel Boulos, Wilson y Clauson, 2018). La separación puede ser suave (soft fork), es decir, se crea una cadena más corta que es descartada rápidamente, o dura (hard fork), es decir, se crea una nueva blockchain con los nodos de la versión antigua (Lin y Liao, 2017), como es el ejemplo de Ethereum y Ethereum Classic (Kaal, 2017).

Para solucionar los problemas de fork se han desarrollado nuevos proyectos, como SegregatedWitness, SegWit (Olsen, Battiston, Caldarelli, Golub Nikulin e Ivliev, 2018), los que buscan mejorar los protocolos de consenso y así solucionar las dificultades existentes, como el Proof of Activity (PoA), basado en el Proof of Work (PoW) y el Proof of Stake (PoS); el Chain of Activity, basado en el PoA (Cocco, Pinna y Marchesi, 2017). PoS se basa en los participantes con mayor cantidad de tokens como los más interesados en 
mantener la integridad de la red, al ser quienes han apostado más por esta plataforma (Meng, Tischhauser, Wang, Wang y Han, 2018), por lo que tienen mayor cantidad de votos.

\subsection{Compromiso entre escalabilidad y velocidad}

La compensación entre escalabilidad y velocidad, tiempo de transacciones, es otro problema de la plataforma que se debe solucionar. Para mejorar la escalabilidad en la plataforma de blockchain se desarrollan implementaciones como Simplified Payment Verification (SPV), que procura reducir la cantidad de datos almacenados al utilizar solo el mensaje de cabecera del bloque (Lin y Liao, 2017).

Si comparamos el tiempo de una transacción de pago, las confirmaciones de las transacciones realizadas con tarjeta de crédito demoran entre dos y tres días. Con bitcoin esta verificación demora solo una hora (Lin y Liao, 2017) y la generación del bloque demora un minuto (Anh Dinh, Liu, Zhang, Chen, Chin Ooi y Wang, 2018). El ejemplo mencionado nos muestra con gran facilidad cómo la tecnología blockchain puede reducir los tiempos de transacciones; en una de las transacciones más comunes y simples como es el pago con tarjeta de crédito, podemos proyectar los enormes beneficios en reducción de tiempos y por lo tanto de dinero que genera el utilizar esta tecnología en transacciones más complejas, sobre todo en las que se tiene mayor cantidad de entidades involucradas.

Otras propuestas buscan reducir la carga de procesamiento y utilizar sensores en dispositivos móviles (Zhang, Xue y Huang, 2016), así como expandir la aplicación del blockchain al sector salud, recopilando información de los pacientes. El planteamiento nos permite acercarnos a una situación en la que el mismo paciente puede registrar sus características físicoquímicas, como presión arterial, nivel de glucosa, entre otros; mediante un dispositivo loT, el equipo envía los datos al nodo más cercado para la creación del bloque pertinente en la cadena y así el doctor puede revisar el historial de todas las mediciones sin necesidad de estar presente en la misma medición.

\section{Beneficios de la transparencia y confianza de blockchain en diversos sectores}

Los beneficios de transparencia y confianza que nos ofrece la tecnología blockchain tienen una gran versatilidad de aplicaciones. Como hemos visto con la cadena de suministro, de la misma forma se puede aplicar a la cadena de demanda al crear bloques con información de los gustos y preferencias de los clientes en la misma tienda, la que podrá ser compartida con los distribuidores, fabricantes y todos en la red.

\subsection{Aplicaciones en sostenibilidad}

En relación con la sostenibilidad se están desarrollando varias propuestas, como el uso de la plataforma blockchain enlazada con el esquema de comercialización de emisiones 
(ETS o Emission Trading Scheme), en la industria textil, por ser esta responsable de aproximadamente $10 \%$ de las emisiones de carbono (World Wildlife Fund, 2013). El esquema ETS se basa en el seguimiento de la norma y límites estipulados por las autoridades $\mathrm{y}$, combinado con la tecnología blockchain, se puede manejar un token basado en las unidades permitidas de carbono que distribuye la autoridad del gobierno correspondiente a las empresas que lo utilizan para la fabricación, empaque y transporte de sus bienes, además de comercializar estos tokens entre estas (Fu, Shu y Liu, 2018). Así se evita la falsificación, fraude o sobornos relacionados con las emisiones de carbono de las compañías, y se controla de forma más eficiente la contaminación en el planeta.

También podemos apreciar iniciativas relacionadas con la eficiencia del uso de la electricidad y la comercialización del excedente de esta por consumidores que generen energía renovable (Huang, Choi, Lee, Jeon, Kim, Park y Park, 2017). Uno de los mayores problemas es el uso de energías no renovables. En varios países desarrollados las familias están utilizando paneles solares, molinos de viento, entre otros; sin embargo, la energía que ellos generan y no utilizan se pierde. Lo que nos indica la propuesta es la posibilidad de aprovechar esa energía generada para venderla a otras familias o entidades que no la generaron suficientemente o que no la pueden generar, y de esta forma emplear menos energía no renovable.

\subsection{Aplicaciones en el sector financiero}

Dentro del sector financiero se han realizado avances con Smart Contracts, buscando reducir tiempos y costos. La gestión de los contratos se puede realizar con la tecnología blockchain si separamos la elaboración de los términos del contrato de su ejecución, de forma que la gestión del contrato pueda darse de forma imparcial (Egelund-Müller, Elsman, Henglein y Ross, 2017). Los Smart Contracts pueden encargarse de la parte de la ejecución. Al ser programados con las reglas del negocio, se ejecutarán automáticamente cuando ciertas condiciones se cumplan, como pagos a una fecha determinada, cobros de algún instrumento financiero, emisión de facturas, entre otros.

\subsection{Aplicaciones en lotería}

Otras aplicaciones interesantes para el uso de la tecnología blockchain pueden dirigirse al manejo de la lotería en Smart Cities, FairLotto, y así garantizar la equidad, transparencia y privacidad del modelo (Liao y Wang, 2017), dando mayor confianza al consumidor en torno de las probabilidades que pueda tener su boleto de lotería.

\subsection{Aplicaciones en voto electrónico}

También se tienen modelos orientados al voto electrónico, eliminando el fraude de este (Hanifatunnisa y Rahardjo, 2017). La transparencia y confiabilidad que nos ofrece un 
sistema distribuido es ideal para la gestión de votaciones electrónicas (Khan, Arshad y Khan, 2018). Las propuestas mencionadas con blockchain van a revolucionar todo tipo de elecciones al devolver al votante la confianza en el sistema de votación, especialmente en países en desarrollo donde esta confianza es menor. Asimismo, eliminará la posibilidad de corrupción, sobornos y manipulación de los votos. Al tener todos los nodos en la red acceso a la información en la cadena de bloques, es imposible manipularla o generar bloques falsos.

\subsection{Aplicaciones en educación}

Se pueden ver propuestas en el sector de educación como EduCTX, basado en un sistema distribuido de punto a punto que maneja y controla los ECTS (Sistema Europeo de Acumulación y Transito de Créditos por sus siglas en inglés) de forma confiable y transparente (Turkanovic, Hölbl, Kosic, Hericko y Kamisalic, 2018). Todas las entidades de educación superior controlan el progreso de los alumnos con base en las notas y los créditos que se les otorgan al aprobar los diferentes cursos. Estos créditos están asociados con el número de horas necesarias para cada curso; en el Perú es una relación de las horas de dictado de clase, es decir, horas presenciales. En Europa los créditos ECTS están basados en la cantidad de horas que el alumno necesita dedicarle al curso para aprobarlo de manera satisfactoria; estas horas pueden ser horas de dictado de clase, de estudio, de visitas a empresas, de elaboración de proyectos, de investigación, entre otras. El espíritu de los créditos ECTS es estandarizar el avance curricular y nivel de educación que tiene cada estudiante, de forma que el grado otorgado por una institución en un país tiene el mismo valor al tener la misma cantidad de créditos ECTS en cualquier país de Europa.

Podemos apreciar la gran ventaja de manejar la información de los créditos ECTS en una plataforma blockchain, donde los bloques son inmutables una vez creados, e imposibles de falsificar o adulterar. Así se reduce la falsificación de certificados o títulos profesionales. La Universidad de Nicosia es la primera en utilizar la tecnología blockchain para manejar los certificados de sus estudiantes (Sharples y Domingue, 2016). Otros planteamientos buscan integrar el registro de las competencias adquiridas por los alumnos y los resultados del aprendizaje o learning outcomes que obtienen. Gran cantidad de instituciones de estudios superiores manejan un currículo basado en competencias, las cuales son medidas a través de evaluaciones como exámenes, trabajos o exposiciones. En este punto se han encontrado algunos problemas en la automatización de la medición de los trabajos o exámenes en Smart Contracts, dado que estos pueden contener partes subjetivas que necesitan de una persona para que defina el resultado (Chen, Xu, Lu y Chen, 2018). Por ello, no podemos tener un sistema de medición de competencias del alumno completamente automatizado con la tecnología blockchain, sino que debe ser un sistema semiautomático donde exista la interacción de personas en la calificación de los trabajos de los alumnos. 


\section{Conclusiones}

Observamos con claridad que existen muchas formas de aprovechamiento de la tecnología blockchain, especialmente al incrementar la confianza en las diferentes entidades involucradas en una misma la cadena de suministro a través de la transparencia que brinda de la información. De esta misma forma podríamos hablar de la cadena de demanda, es decir, complementar la información que se maneja en la cadena de suministro con los gustos y preferencias de los consumidores, lo que sería de gran ayuda para los mayoristas, distribuidores, operadores logísticos, fabricantes, etcétera.

Al revisar la literatura podemos encontrar gran cantidad de investigaciones académicas así como proyectos piloto que enfatizan la enorme utilidad de la tecnología blockchain para mantener la integridad de la información desde las entidades productoras hasta el consumidor final. Sin embargo, debemos identificar que la cadena de demanda, que va en sentido inverso, del consumidor final a la entidad productora, es tan importante para las empresas como la cadena de suministro.

La cadena de suministro puede aprovechar la tecnología blockchain para evitar problemas de ruptura de stock, en planificación, y contiene información muy valiosa para las áreas de marketing, desarrollo de producto y diseño de servicios, al contener el detalle de los gustos y preferencias del consumidor, información que puede ser identificada en las tiendas minoristas, pero difícilmente regresa con el mismo detalle a través de toda la cadena hasta la fábrica.

También hemos evidenciado los problemas que aún tiene esta tecnología en cuanto a riesgo en seguridad de los Smart Contracts y la importancia de programarlos a prueba de fallas o de ataques maliciosos. Asimismo, hemos expuesto los avances de la combinación de la tecnología blockchain con dispositivos loT, los cuales carecen de grandes capacidades de procesamiento y muchos de ellos, como los sensores, tienen poca capacidad de almacenamiento de información.

Conforme la tecnología de blockchain avanza podemos descubrir diferentes versiones de algunas de las plataformas que en la actualidad son más de 17 (Shanley, 2017), lo que nos lleva a problemas fork, cuando la cadena se divide en dos cadenas de bloques al tener protocolos de conceso diferenciados, y puede llevar a separar la red en dos plataformas diferenciadas de blockchain, como es el caso de Ethereum y Ethereum Classic (Kaal, 2017).

La tecnología blockchain nos ofrece un nuevo universo, donde la confianza entre entidades no es necesaria al estar garantizada por la plataforma con la verificación de los bloques antes de ser aceptados en la cadena. También es importante resaltar la transparencia: los nodos pueden acceder a toda la información en la red. Finalmente, los Smart Contracts nos facilitan nuevos modelos de negocios con transacciones y contratos totalmente automatizados sin la necesidad de intervención humana. 


\section{Referencias}

Anh Dinh, T., Liu, R., Zhang, M., Chen, G., Chin Ooi, B., y Wang, J. (2018, Julio). Untangling Blockchain: A Data Processing View of Blockchain Systems. IEEE Transactions on Knowledge and Data Engineering, 30(7), 1366-1385. doi:10.1109/TKDE.2017.2781227.

Atzei, N., Bartoletti, M. y Cimoli, T. (2017). A Survey of Attacks on Ethereum Smart Contracts (SoK). 6th International Conference on Principles of Security and Trust (pp. 164-186). Uppsala, Sweden: Spriger.

Beninger, P. (2017). Opportunities for Collaboration at the Interface of Pharmacovigilance and Manufacturing. Clinical Therapeutics, 39(4), 702-712. Recuperado de: http://dx.doi. org/10.1016/j.clinthera.2017.03.010.

Casey, M. y Wong, P. (2017). Global supply chains are about to get better, thanks to blockchain. Harvard Business Review, 13.

Chen, G., Xu, B., Lu, M. y Chen, N.-S. (2018). Exploring blockchain technology and its potential applications for education. Smart Learning Environments, 5(1), 1-10.

Christidis, K. y Devetsikiotis, M. (2016). Blockchains and smart contracts for the internet of things. IEEE Access, 4, 2292-2303. doi:10.1109/ACCESS.2016.2566339.

Cocco, L., Pinna, A. y Marchesi, M. (2017). Banking on Blockchain: Costs Savings Thanks to the Blockchain Technology. Future internet, 9(25), 1-20.

Egelund-Müller, B., Elsman, M., Henglein, F. y Ross, O. (2017). Automated Execution of Financial Contracts on Blockchains. Business y Information Systems Engineering.

Engelenburg, S., Janssen, M. y Klievink, B. (2017). Design of a software architecture supporting business-to-government information sharing to improve public safety and security. Journal of Intelligent information systems. doi:10.1007/s10844-017-0478-z.

Faye, P. (2017). Use of Blockchain Technology in Agribusiness: Transparency and Monitoring in Agricultural Trade. 4th International Conference on Management Science and Management Innovation. 31, pp. 38-40. Suzhou, China: AEBMR-Advances in Economics Business and Management Research.

Firica, O. (Octubre de 2017). Blockchain technology: Promises and realities of the year 2017. QualityAccess to Success, 18(S3), 51-58.

Fu, B., Shu, Z. y Liu, X. (2018). Blockchain Enhanced Emission Trading Framework in Fashion Apparel Manufacturing Industry. Sustainability, 10(1105), 1-19. 
Hanifatunnisa, R. y Rahardjo, B. (2017). Blockchain Based E-Voting Recording System Design. 11th International Conference on Telecomunication Systems Services and Applications. Lombok, Indonesia: IEEE.

Hwang, J., Choi, M.-I., Lee, T., Jeon, S., Kim, S., Park, S. y Park, S. (2017). Energy Prosumer Business Model Using Blockchain System to Ensure Transparency and Safety. 4th International Conference on Power and Energy Systems Engineering (pp. 194-198). Berlin, Alemania: Elsevier.

Kaal, W. (2017). Crypto Transaction Dispute Resolution. The Business Lawyer, 109-152.

Kamel Boulos, M. N., Wilson, J.T.y Clauson, K. A. (2018). Geospatial blockchain: promises, challenges, and scenarios in health and healthcare. International Journal of Health Geographics, 17-25.

Khan, K., Arshad, J. y Khan, M. (2018). Secure Digital Voting System Based on Blockchain Technology. International Journal of Electronic Government Research, 14(1), 53-62.

Kshetri, N. (2018). Blockchain's roles in meeting key supply chain management objectives. International Journal of Information Management, 39, 80-89.

Liao, D.-Y. y Wang, X. (2017). Design of A Blockchain-based Lottery System for Smart Cities Applications. 3rd International Conference on Collaboration and Internet Computing (pp. 275-282). Newark, California: IEEE.

Lin, I.-C. y Liao, T.-C. (2017). A Survey of Blockchain Security Issues and Challenges. International Journal of Network Security, 19(5), 653-659.

Madhwal, Y. y Panfilov, P. B. (2017). Blockchain and supply chain management: Aircrafts' parts' business case. Vienna:28th DAAAM International Symposium on intelligent manufacturing and automation. doi:10.2507/28th.daaam.proceedings.146.

Meng, W., Tischhauser, E. W., Wang, Q., Wang, Y. y Han, J. (2018). When Intrusion Detection Meets Blockchain Technology: A Review. IEEE Access, 6, 10179-10188.

Neisse, R., Steri, G. y Nai-Fovino, I. (2017). A Blockchain-based Approach for Data Accountability and Provenance Tracking. 12th International Conference on availability, reliability and security. Calabria, Italy: ACM.

Olsen, R., Battiston, S., Caldarelli, G., Golub, A., Nikulin, M. y Ivliev, S. (2018). Case study of Lykke exchange: architecture and outlook. Journal of Risk Finance, SI, 26-38.

Parra Moyano, J. y Ross, O. (2017). KYC Optimization Using Distributed Ledger Technology. Business Infromation Systems Engineering, 59(6), 411-423. 
Pincheira Caro, M., Salek Ali, M., Vecchio, M. y Giaffreda, R. (2018). Blockchain-based Traceability in Agri-Food Supply Chain Management: A Practical Implementation. 2018 IoT Vertical and Topical Summit on Agriculture (págs. 1-4). Tuscany: IEEE.

Qu, C., Tao, M., Zhang, J., Hong, X. y Yuan, R. (junio de 2018). Blockchain Based Credibility Verification Method for loT Entities. Security and Communication Networks, 2018, 1-11. doi:doi. org/10.1155/2018/7817614.

Sander, F., Semeijn, J. y Mahr, D. (2018). The acceptance of blockchain technology in meat traceability and transparency. British Food Journal, 120(9), 2066-2079.

Shanley, A. (2017). Could Blockchain improve pharmaceutical supply chain security? Pharmaceutical Technology, 34-39.

Sharples, M. y Domingue, J. (2016). The Blockchain and Kudos: A Distributed System for Educational Record, Reputation and Reward. European Conference on Technology Enhanced Learning Adaptive and Adaptable Learning (pp. 490-496). Lyon, France: Springer.

Sun, J., Yan, J. y Zhang, K. Z. (2016). Blockchain-based sharing services: What blockchain technology can contribute to smart cities. Financial Innovation, 2(26), 1-9.

Tian, F. (2017). A Supply Chain Traceability System for Food Safety Based on HACCP, Blockchain and Internet of Things. 14th Internatonal Conference on Services Systems and Services Management. Dalian, China: International Conference on Service Systems and Service Management.

Toyoda, K., Sasase, I., Ohtsuki, T. y Mathiopoulos, P. (2017). A Novel Blockchain-Based Product Ownership Management System (POMS) for Anti-Counterfeits in the Post Supply Chain. IEEE Access, 5, 17465-17477. doi:10.1109/ACCESS.2017.2720760.

Turkanovic, M., Hölbl, M., Kosic, K., Hericko, M. y Kamisalic, A. (2018). EduCTX: A Blockchain-Based Higher Education Credit Plataform. IEEE Access, 6, 5112-5126.

World Wildlife Fund (2013). Cutting Cotton Carbon Emissions:Findings from Warangal, India. WWF Report. Recuperado de: http://www.indiaenvironmentportal.org.in/files/file/cotton_ carbon_emission.pdf.

Wu, H., Li, Z., King, B., Miled, Z. B., Wassick, J. y Tazelaar, J. (2017). A Distributed ledger for supply chain physical distribution visibility. Information 8(137), 1-18. doi:10.3390/info8040137.

Xu, J. J. (2016). Are blockchains immune to all malicious attacks? Financial Innovation, 2(25), 1-9. 
Yeow, A. (2018). Bitnodes. Recuperado de: earn.com: https://bitnodes.earn.com/.

Yeow, K., Gani, A., Rodrigues, J., Ahmad, R. y Ko, K. (2017). Decentralized Consensus for Edge-Centric Internet of Things: A Review, Taxonomy, and Research Issues. IEEE Access.

Zhang, J., Xue, N. y Huang, X. (2016). A secure system for pervasive social network-based healthcare. IEEE Access, 4, 9239-9250. doi:10.1109/ACCESS.2016.2645904. 
